\title{
Eriodictyol can modulate cellular auxin gradients to efficiently promote in vitro cotton fibre development
}

\author{
Anam Qadir Khan', Zhonghua Li', Muhammad Mahmood Ahmed ${ }^{1,2}$, Pengcheng Wang ${ }^{1}$, Xianlong Zhang ${ }^{1}$ and \\ Lili Tu ${ }^{1 *}$
}

\begin{abstract}
Background: Flavonoids have essential roles in flower pigmentation, fibre development and disease resistance in cotton. Previous studies show that accumulation of naringenin in developing cotton fibres significantly affects fibre growth. This study focused on determining the effects of the flavonoids naringenin, dihydrokaempferol, dihydroquerectin and eriodictyol on fibre development in an in vitro system.

Results: $20 \mu \mathrm{M}$ eriodictyol treatment produced a maximum fibre growth, in terms of fibre length and total fibre units. To gain insight into the associated transcriptional regulatory networks, RNA-seq analysis was performed on eriodictyoltreated elongated fibres, and computational analysis of differentially expressed genes revealed that carbohydrate metabolism and phytohormone signaling pathways were differentially modulated. Eriodictyol treatment also promoted the biosynthesis of quercetin and dihydroquerectin in ovules and elongating fibres through enhanced expression of genes encoding chalcone isomerase, chalcone synthase and flavanone 3-hydroxylase. In addition, auxin biosynthesis and signaling pathway genes were differentially expressed in eriodictyol-driven in vitro fibre elongation. In absence of auxin, eriodictyol predominantly enhanced fibre growth when the localized auxin gradient was disrupted by the auxin transport inhibitor, triiodobenzoic acid.
\end{abstract}

Conclusion: Eriodictyol was found to significantly enhance fibre development through accumulating and maintaining the temporal auxin gradient in developing unicellular cotton fibres.

Keywords: Auxin transport, Cotton fibre, Eriodictyol, Fibre development, Flavonoids, Flavonoid biosynthesis

\section{Background}

Flavonoids are a diverse group of secondary metabolites that are commonly found in nature and play various roles that assist plants in multiple biological activities $[1,2]$. They have been proven beneficial to various crop plants by providing resistance against pathogen infections [3]. However, the most visible impact of flavonoids is their potential to produce pigmentation in flowers, fruits and seeds [4]. Flavonoid biosynthesis leads to the production of six different classes of compounds amongst which the anthocyanins, responsible for pigmentation in flowers, are widespread in plants [5]. In cotton,

\footnotetext{
* Correspondence: lilitu@mail.hzau.edu.cn

${ }^{1}$ National Key Laboratory of Crop Genetic Improvement, Huazhong

Agricultural University 430070, Wuhan, Hubei, People's Republic of China

Full list of author information is available at the end of the article
}

approximately 52 flavonoids are involved in modulating fibre development, pigmentation and regulating other functions [6]. Color variations in cotton flower petals have also shown to be influenced by flavonoid accumulation [7]. Silencing of FLAVANONE 3-HYDROXYLASE (F3H; TT6) in cotton blocked flavonoid biosynthesis which changed the flower petal color from cream to white, due to reduced anthocyanin content [7].

Flavonoids are considered crucial in modulating pigmentation in naturally colored cotton fibres and recently, many studies have explored the genetic and metabolic basis of fibre pigmentation using biotechnological tools. A study reported that general flavonoid biosynthesis pathway genes, such as CHALCONE SYNTHASE (CHS), FLAVANONE 3'-HYDROXYLASE (F3'H) and FLAVANONE 3'5'HYDROXYLASE $\left(F 3^{\prime} 5^{\prime} H\right)$, were differentially expressed in 
brown cotton fibres compared to white cotton fibres [8]. Combined transcriptomic and metabolomic analyses showed that proanthocyanins (PAs) such as gallocatechin and catechin were abundantly present in brown cotton fibres because flavonoid biosynthesis pathway genes were highly expressed [9]. Another study reported that a TT2type MYB transcription factor modulated proanthocynidin biosynthesis through interacting with promoter elements to enhance the transcriptional activity of two downstream flavonoid biosynthesis genes ANTHOCYANIDIN REDUCTASE (ANR) and LEUCO-ANTHOCYANIDIN REDUCTASE (LAR) [10]. The overexpression of GhTT2-3A during secondary cell wall synthesis resulted in brown cotton fibres [11]. Although flavonoid biosynthesis in pigmented fibres is well established in cotton, engineering of this pathway for the production of higher-quality cotton fibres has not been well exploited.

Over the last decade, many researchers have emphasized the putative role of the flavonoid metabolic pathway in the modulation of anthocyanin and PA biosynthesis in cotton $[8,12]$. Interestingly, transcriptomic analyses have suggested a potential involvement of flavonoids in fibre development, since various flavonoid biosynthesis genes have been found to be differentially expressed in developing fibres [13, 14]. Similarly, comparative transcriptome and metabolome analyses have revealed a significant abundance of flavonoids in elongating fibres of G. barbadense, a cultivated tetraploid cotton that produces high-quality fibre $[15,16]$. However, the critical role of flavonoids during fibre development remained obscure until a study demonstrated that silencing $F 3 H$ suppressed fibre elongation due to the accumulation of naringenin (NAR) [17].

Flavonoids have also been considered to be modulators of auxin signaling since they may be distributed asymmetrically, thus affecting auxin transport by enhancing localized accumulation [18, 19]. They have been demonstrated to interact with auxin transport components through a class of transcription factors, WRKY, which ultimately regulate root development in Arabidopsis [20]. Recently, a review summarized compelling evidence that flavonoids mediate phytohormone signaling and growth responses in plants [21]. Flavonoids may modulate auxin signaling and transport through their ability to scavenge reactive oxygen species (ROS) [22] and can affect a wide range of proteins [18]. Although flavonoids were once considered to inhibit auxin signaling, in reality, they establish an auxin gradient in single cells [23]. However, the link among flavonoids, auxin and growth has not been extended to unicellular cotton fibre cells in vitro or in planta.

Several developmental processes such as morphogenesis, hormone signaling and ROS homeostasis in the perspective of fibre biology have been studied using cotton ovule culture $[17,24-26]$. The critical role of flavonoids in cotton fiber development was also witnessed using the cotton ovule culture. Interestingly, endogenous levels of two flavanols (eriodictyol, ERI and naringenin, NAR), two dihydroflavanols (dihydrokaempferol, DHK and dihydroquerectin, DHQ) and two flavanones (kaempferol, $\mathrm{K}$ and quercetin, $\mathrm{Q}$ ), were found to be influenced by $\mathrm{F} 3 \mathrm{H}$ activity in the $\mathrm{F} 3 \mathrm{H}$-transgenic cotton lines, and NAR was found to inhibit fibre development [17]. To further dissect the molecular mechanisms underlying the role of flavonoids, we here examined the effects of six different, exogenously applied flavonoids on cotton fibre development. We determined the optimal concentration at which maximal in vitro fibre growth was observed and found that ERI significantly promotes fibre development. RNA-seq analysis was performed to gain insights into the transcriptional networks modulated by ERI treatment that could promote fibre growth in ovule culture. This study suggests the potential of the flavonoid ERI in promoting fibre growth through effects on fibre initiation and elongation.

\section{Results}

\section{Exogenous ERI promotes fibre development in vitro}

Genetic engineering of the flavonoid metabolic pathway is considered promising for improving fibre development. Silencing F3H leads to NAR accumulation, which is negatively associated with fibre development, while its overexpression does significantly affect fibre phenotype [17]. It is therefore of significant interest to understand the expression pattern of flavonoid pathway genes during in vitro cotton fibre development, and how this is linked to growth effects. An ovule culture assay was performed in which 6 different flavonoids (ERI, NAR, DHQ, DHK, Q and K) were supplemented to the BT medium at a concentration of $10 \mu \mathrm{M}$. Fibre development in response to flavonoid treatment was determined by measuring fibre length and the total fibre unit (TFU) compared with controls. After 10 days of culture, the fibre phenotype of cultured ovules was observed to be differentially influenced by the six flavonoids (Fig. 1a). NAR and DHK both suppressed fibre development in terms of fibre length and TFU (Fig. 1b-c). Treatment with two flavanols ( $\mathrm{Q}$ and $\mathrm{K}$ ) did not significantly affect fibre development, and therefore were not investigated further (Fig. 1b-c). Effects on fibre length and TFU were investigated following in vitro ovule treatment with ERI and DHQ, both of which are produced through the enzymatic action of F3'H on NAR and DHK respectively (Fig. 1c). ERI efficiently promoted fibre development, which suggests that $F 3^{\prime} H$ activity may have a role in determining fibre growth dynamics during early fibre development. 
a

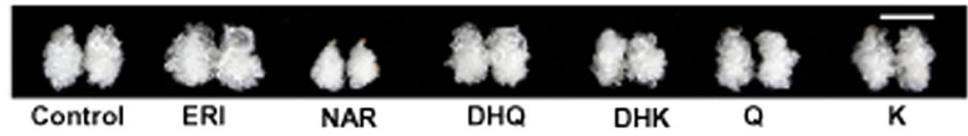

b
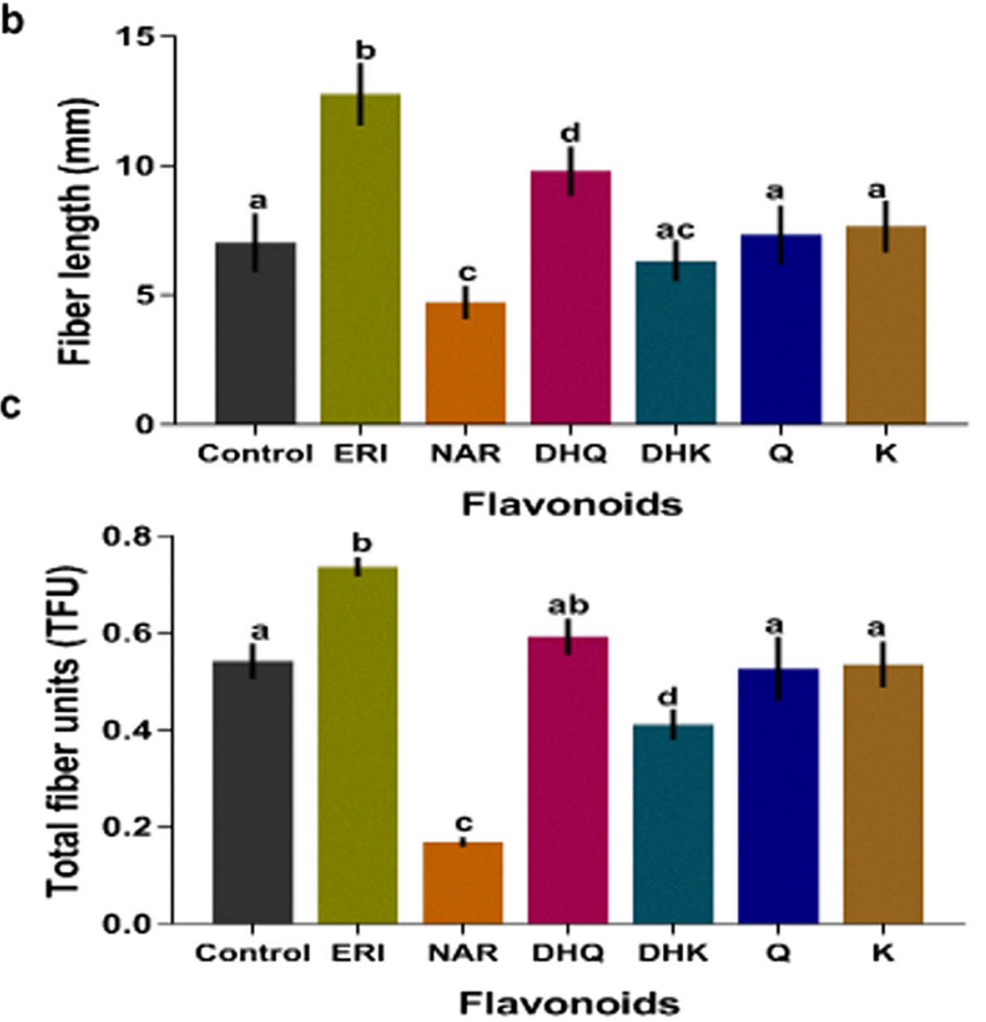

Fig. 1 Exogenous ERI treatment promotes in vitro fibre development. (a) The fibre phenotype of 10-day-cultured ovules without or with different flavonoids treatments, i.e., control, ERI, NAR, DHQ, DHK, Q and K. The white line represents the scale bar, $5 \mathrm{~mm}$. (b) Fibre length after 10 days of culture from in response to different flavonoid treatments. (c) Total fibre unit (TFU) in 10-day-cultured ovules in response to different flavonoids. For b-c, error bars indicate SD for three repeats, and lowercase letters present significant differences between the treatments

ERI promotes the initiation and elongation stages of fibre development

In vitro ovule culture assays provided an opportunity to test the effect of flavonoids on fibre development. We determined the optimum ERI concentration needed for maximum fibre growth in vitro and compared the responses of NAR, DHK and DHQ in the presence of growth-regulating hormones, i.e., indole acetic acid (IAA) and gibberellic acid $\left(\mathrm{GA}_{3}\right)$. To achieve this goal, a series of ovule culture experiments were therefore performed with the four flavonoids (ERI, NAR, DHQ and DHK) each at six different concentrations $(0,2,5,10,20$ and $40 \mu \mathrm{M})$.

After 10 days of culture, ERI-treated ovules showed more vigorous and fluffier fibre growth when supplemented with 5-40 $\mu \mathrm{M}$ (Fig. 2a). The application of either DHQ or DHK slightly suppressed fibre growth, while no significant differences in fibre development and yield were observed when either 5,10 or $20 \mu \mathrm{M}$ concentrations were applied. DHQ inhibited normal fibre development at 2 and $40 \mu \mathrm{M}$, while DHK inhibited it at 2, 5 and $20 \mu \mathrm{M}$. In contrast ERI showed a concentrationdependent effect on fibre growth. A less consistent pattern was observed for DHK and DHQ, while NAR induced a strong inhibitory effect on fibre length and TFU at $10 \mu \mathrm{M}$, but not at lower or higher concentrations (Fig. 2b-c). Fibre growth was not significantly changed after treatment with ERI at $0 \mu \mathrm{M}$ and $2 \mu \mathrm{M}$ while it was observed maximum at 20 and $40 \mu \mathrm{M}$ concentrations (Fig. 2b-c). Overall, ERI significantly promoted the fibre development in terms of length and TFU at $20 \mu \mathrm{M}$ concentration, which was used for subsequent experiments.

To determine whether ERI has an effect on fibre initiation, ovules cultured for 1 day in BT medium were supplemented with $0 \mu \mathrm{M}$ (Control_1D) and $20 \mu \mathrm{M}$ ERI (ERI_1D) and examined for fibre density. 


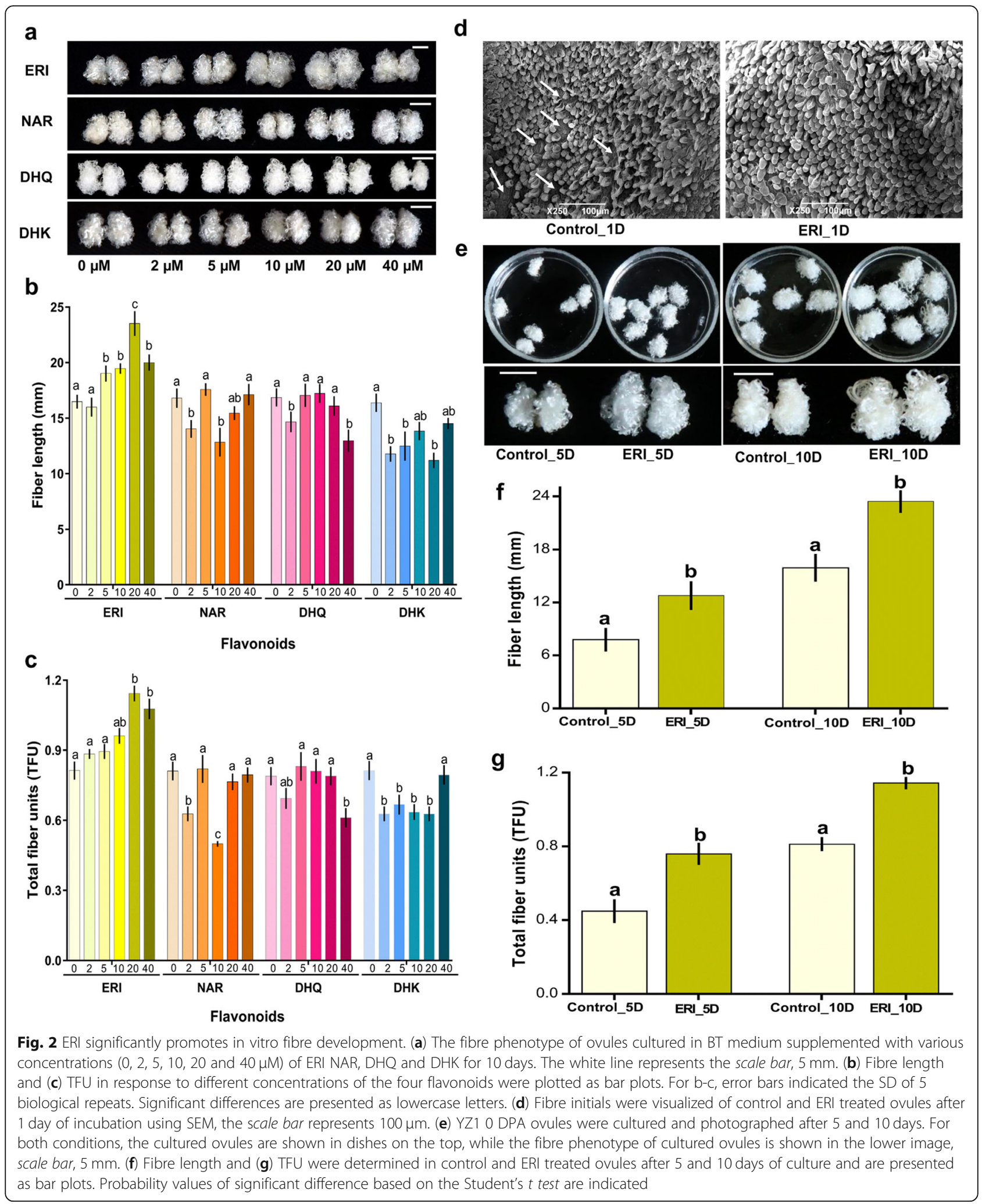

Scanning electron microscopic (SEM) images showed normal differentiation and initiation of fibre cells from the ovule epidermal cells in Control_1D, whereas a greater number of initiating fibres and faster elongation were observed in ovule epidermal cells in ERI_1D (Fig. 2d). Therefore, $20 \mu \mathrm{M}$ ERI application 
promotes fibre initiation and early elongation within 1 day of application.

Similarly, both early ( 5 days) and late (10 days) stages of fibre elongation were analyzed for fibre yield to determine whether ERI could significantly affect fibre elongation over a longer developmental period. Ovules showing representative fibre phenotypes from the four treatments (Control_5D, ERI_5D, Control_10D and ERI_10D) are shown in (Fig. 2e). Both fibre length and TFU were significantly enhanced in ERI_5D and ERI_ 10D ovules compared with Control_5D and Control_ 10D, respectively (Fig. 2f-g). These findings clearly showed that fibre development was strongly promoted by $20 \mu \mathrm{M}$ ERI during fibre initiation and early and late fibre elongation stages.

\section{RNA-seq analysis}

To gain insight into transcriptional regulatory networks in response to ERI treatment during initiation and early and late fibre elongation stages, we performed whole transcriptome RNA sequencing analysis for tissues collected from Control and ERI-treated samples after 1, 5 and 10 days (designated as Control_1D, ERI_1D, Control_5D, ERI_5D, Control_10D and ERI_10D respectively). Ovules from 1-day-cultured samples and fibres from 5 and 10-days-cultured samples were collected as three biological replicates, and transcriptome libraries were constructed for 18 samples, i.e., 2 treatments (Control, ERI) each at 3 stages $(1,5,10 \mathrm{DPA})$ with 3 replications. Approximately 72.4-74.7 million clean paired-end reads were obtained after trimming adapter sequences and filtering out low-quality reads, and 87.47 - 92.33\% reads of these reads were successfully aligned to the $G$. hirsutum $c v$. TM-1 genome [27]. Of these, a major proportion (60.12 - 67.44\%) accounted for uniquely mapped reads, while $22.57-29.07 \%$ were mapped to multiple sites (Additional file 1: Table S1).

The transcriptional similarity was also ascertained through hierarchical clustering, which distinctively assigned all samples into two major clusters. Cluster I contained replicate samples of Control_1D and ERI_1D while cluster II aggregated all early and late elongation stage samples (Fig. 3a). To minimize the dimensionality in the transcriptomic libraries, the data were normalized to principal components (PCs) through principal component analysis (PCA), and the first two PCs were plotted to visualize variability features. The results showed that samples from the initiation (Control_1D and ERI_1D) and elongation stages (Control_5D, Control_10D, ERI_5D and ERI_10D) were separated from each other (Fig. 3b). Likewise, the expression profiles of 18 samples were well related to the hierarchical clustering results (Fig. 3 a-b; Additional file 2: Figure S1). The results showed that all the samples were clustered according to the distinct biological stages, i.e. initiation and elongation, from which they originated (i.e., 1, 5 and 10 days).

Global transcription profiles were determined for each treatment by analyzing expression data using bioinformatics techniques. The paired comparisons showed that 60,839 to 62,099 genes were co-expressed and 1852 to 2479 genes were specifically expressed in three comparisons (Additional file 3: Figure S2). We found that the

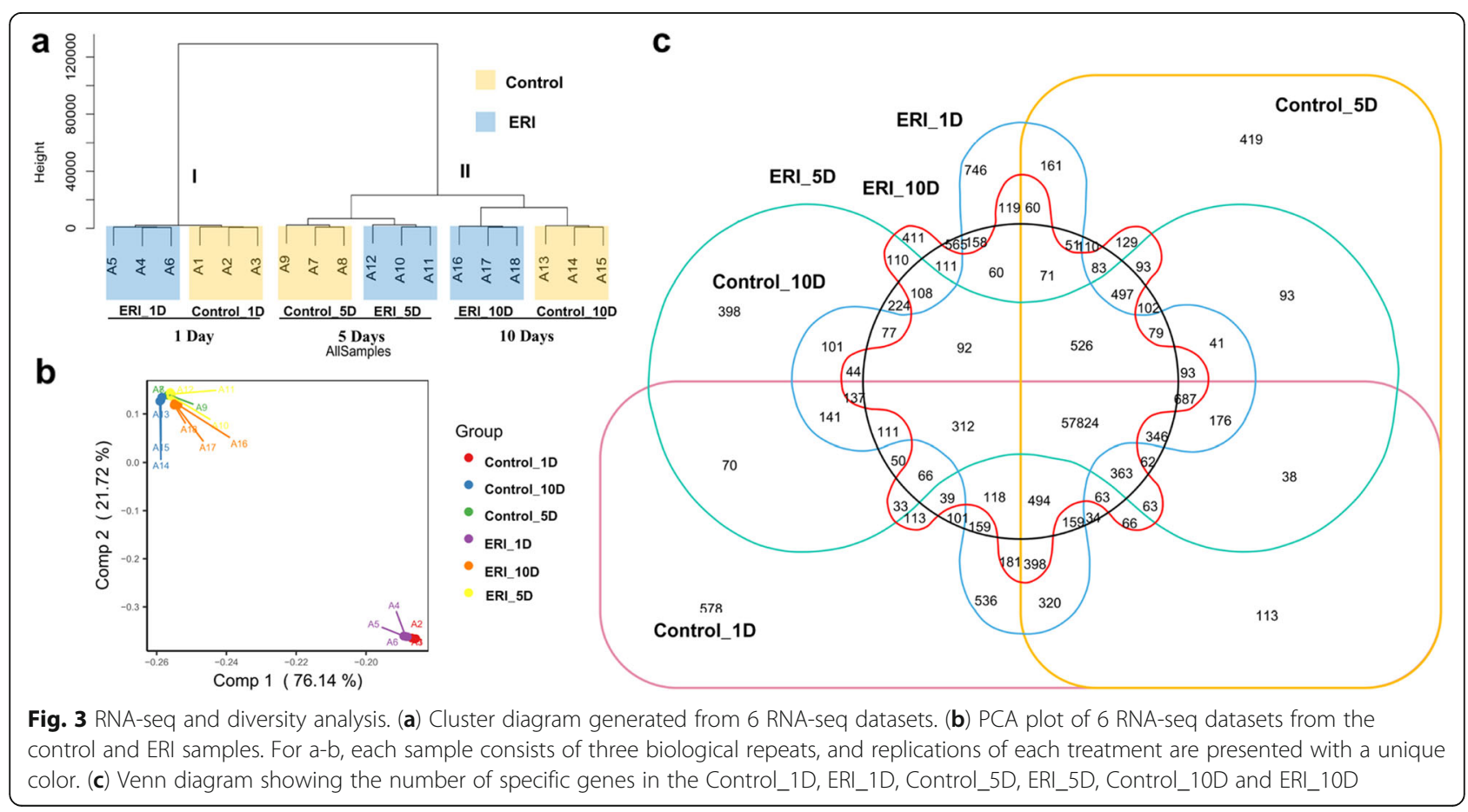


majority of the genes were expressed in both the control $(59,559$ out of 67,713$)$ and ERI $(62,071$ out of 69,901$)$ in all three treatment samples (Additional file 4: Figure S3). There were 57,824 co-expressed genes among 6 samples, while 38,746 genes were co-expressed in 2 to 5 samples (Fig. 3c). Annotated and novel transcribed regions were identified by the cuffcompare utility, which aligns the transcriptome libraries to the TM-1 genome. The results showed that approximately 51,464 to 52,691 annotated genes were identified, i.e. up to $86.98 \%$, while 7712 to 8075 novel transcripts (not annotated) were identified (Additional file 1: Table S1). However, the frequency of expression of annotated and novel transcripts was not significantly different between all Control and ERI paired comparisons (Additional file 5: Figure S4).

\section{Identification and functional analysis of differentially expressed genes (DEGs)}

To explore functional aspects of ERI-based fibre elongation, the number of DEGs was detected using criteria of fold change (FC) ratio $\geq 2$ and false discovery rate $(\mathrm{FDR})<0.05$ to indicate differential expression between control and ERI treatments. Approximately 190 DEGs were detected at fibre initiation (160 up and 30 down regulated between the Control_1D and ERI_1D), 604 at the early elongation stage (245 up and 359 downregulated between the Control_5D and ERI_5D) and 2474 at the late fibre elongation stage (1517 up and 957 down regulated between the Control_ 10D and ERI_10D; Fig. 4a).

Gene Ontology (GO) and Kyoto Encyclopaedia of Genes and Genomes (KEGG) analysis revealed that the most enriched pathways were associated with carbohydrate metabolism (9.67\%), biosynthesis of secondary metabolites (7.22\%) and plant hormonal signal transduction (7.09\%; Fig. 4b). Among various carbohydrate metabolism pathways, ascorbate and aldarate metabolism are considered critical in elongating fibres, since $\mathrm{H}_{2} \mathrm{O}_{2}$ can act as a cell signaling component, triggering various growth-regulating processes [28]. Elongating cotton fibres exhibit ROS homeostasis [25, 29]. To determine whether ascorbate peroxidases are differentially regulated in response to ERI, $\log 2$ values of ERI/ Control ratios were plotted as a heat map (Additional file 6: Figure S5). Our results showed that genes in the ascorbate and aldarate pathway (KEGG00053) were up regulated in ERI_1D (BGI_novel_G009776 and Gh_D10G2055), ERI_5D (Gh_A02G0706, BGI_novel_G000946, Gh_D09G0992, Gh A01G2104, Gh_D05G0229 and Gh_D05G3096) and ERI 10D (BGI_novel_G004748, Gh_A08G1012, Gh_A09G1558, Gh_D08G0759, Gh_D10G1398 and Gh_D12G1841) (Additional file 7: Table S2).

Among the phytohormone pathways, auxin and brassinosteroid signal transduction components were significantly upregulated in the ERI-treated samples. Genes in the auxin signaling cascade were most enriched (64 genes), followed by brassinosteroid signaling components (26 genes) (Fig. 4c). Various genes encode proteins involved in the perception of auxin signaling, such as AUXIN TRANSPORTER PROTEIN1 (AUX1), AUXIN/ INDOLE-3-ACETIC ACID (AUX/IAA), AUXIN RESPONSE FACTORS (ARFS), GH3 and SMALL AUXIN UP RNA (SAUR), which showed differential expression patterns in the ERI samples (Additional file 7: Table S2). These auxin signaling genes were upregulated during the initiation (ERI_1) and late elongation (ERI_10) stages, while $A U X 1, A R F$ and AUXIAA transcripts were less abundant during the early elongation stage (ERI_5) (Fig. 4d). It was interesting to note that $A U X / I A A, G H 3$ and SAUR transcripts, which can regulate auxin-mediated plant growth through cell elongation, were mostly upregulated in ERI_1D, ERI_5D and ERI_10D (Additional file 7: Table S2).

Brassinosteroids (BRs) are another class of growth promoting hormones, and BR signaling is primarily perceived through BRASSINOSTEROID INSENSITIVE1 (BRI1) and BCL2-ANTAGONIST/KILLER1 (BAK1), which are ultimately transduced to activate expression of genes involved in cell wall modification and cell cycling, such as XYLOGLUCAN ENDOTRANSGLUCOSYLASE/ HYDROLASE (TCH4) and CYCLIN-D3 (CYCD3) [30]. We found that BR biosynthesis pathway genes were upregulated in response to ERI treatment (Additional file 8: Figure S6). Similarly, some BR signaling cascade components were also found up-regulated in one or more tissues (Fig. 4e). These results suggested that exogenous ERI modulated both auxin and BR biosynthesis and signaling to promote fibre development.

After determining the KEGG annotations, nine DEGs: TCH4 (Gh_D02G0992, Gh_D05G3030 and Gh_D03G0483), DFR (Gh_D01G0569), LAR (Gh_D01G0977), SAUR (Gh A13G2026), ASCORBATE PEROXIDASE (APX; Gh D08G0759 and Gh_A11G2287) and BAK1 (Gh_D08G2082), were randomly selected to validate the RNA-seq analysis integrity. The RT-qPCR expression was normalized to the expression of the reference gene GhUBQ7 (NCBI XM_ 016855110.1). The results showed that the expression profiles of all tested genes were highly related to the fragments per kilo-base of transcript per million mapped reads (FPKMs) values based on the RNA-seq data (Fig. 5). These findings indicated that the results inferred from the RNAseq data analysis are highly reliable.

\section{The flavonoid pathway actively modulated in response to ERI}

Although other secondary metabolites such as alkaloids and terpenoids have important roles in plants, and are typically species- and tissue-specific, we focused in the current work on flavonoids, since the primary objective of this study was to determine the role of flavonoids and 


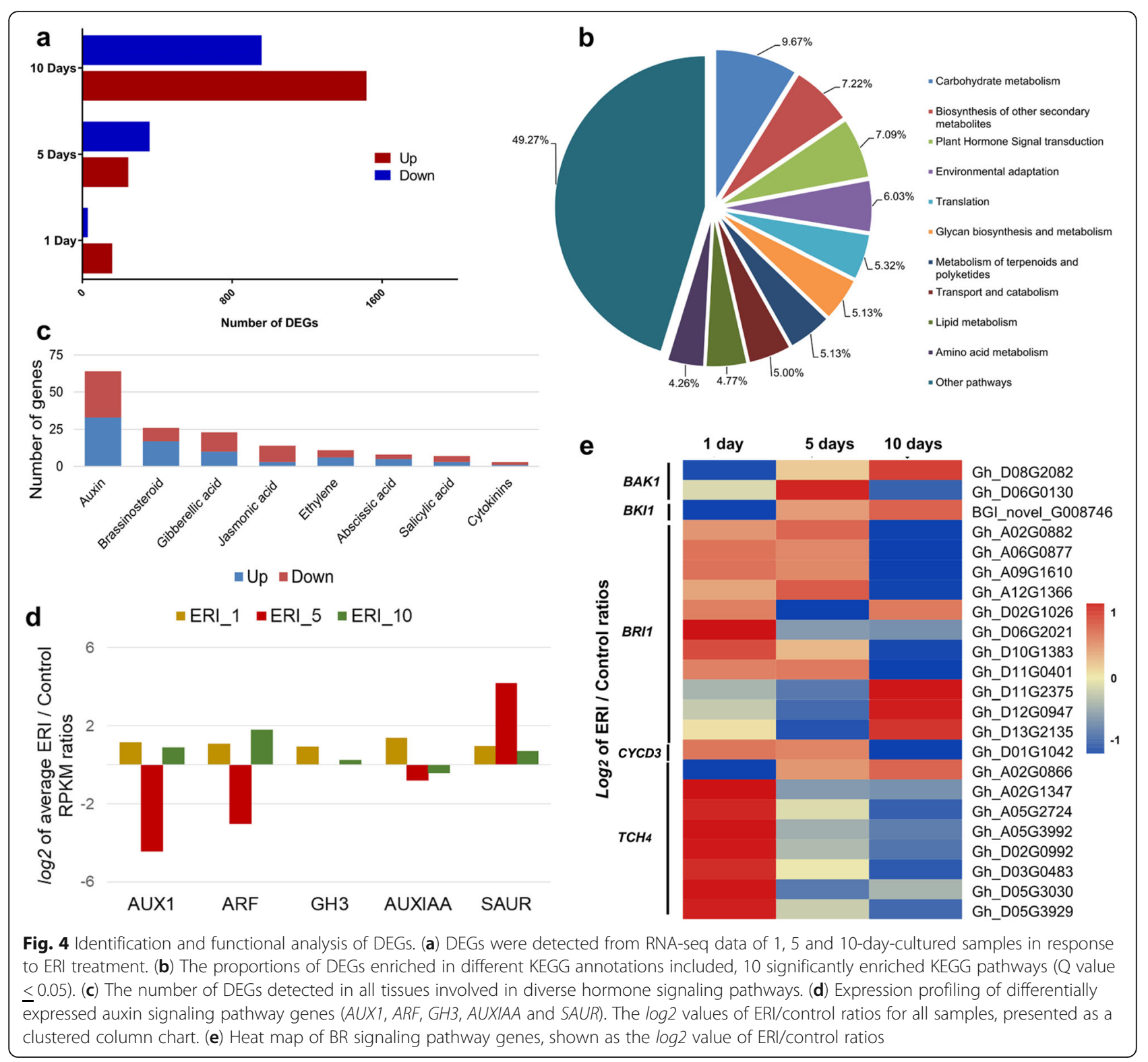

the control of flavonoid biosynthesis in fibre development. Therefore, the expression profiles of flavonoid pathway genes were analyzed. Moreover, we linked flavonoid pathway gene expression to flavonoid content by quantifying flavonoids (ERI, NAR, DHQ, Q, DHK and $\mathrm{K})$ in the six treatment samples.

In the context of flavonoid pathway, the higher $F 3^{\prime} H$ transcript abundance might be expected to result in the synthesis of ERI and DHQ through metabolizing NAR and DHK respectively, since the $F 3 H$ transcripts were more abundant in response to exogenous ERI treatment at day 1, ERI_1D (Fig. 6e). Surprisingly, the endogenous NAR content was not significantly lowered in ERI_1D (Fig. 6g) which might be explained by the augmented production of an alternate $F 3 H$ substrate (ERI) or poor relationship between $F 3 H$ transcript and protein. Likewise, it might also be expected because both CHS and $\mathrm{CHI}$ were upregulated by ERI treatment (Fig. 6). Moreover, the abundance of $F 3^{\prime} H$ transcripts could lead to the catabolism of DHK to DHQ as the endogenous DHQ content was significantly increased, and DHK reduced, even though $F 3 H$ was highly expressed in ERI 1D (Fig. 6a-g). Overall, ERI treatment was associated with the elevated expression of $F 3 H$ and $F 3^{\prime} H$ genes and of the upstream flavonoid biosynthesis genes $C H S$ and CHI.

Despite presumed active biosynthesis of NAR, linked to the up-regulation of $\mathrm{CHS}$ and $\mathrm{CHI}$, it did not accumulate during the initiation and early elongation stages. This may be because the genes capable of metabolizing 


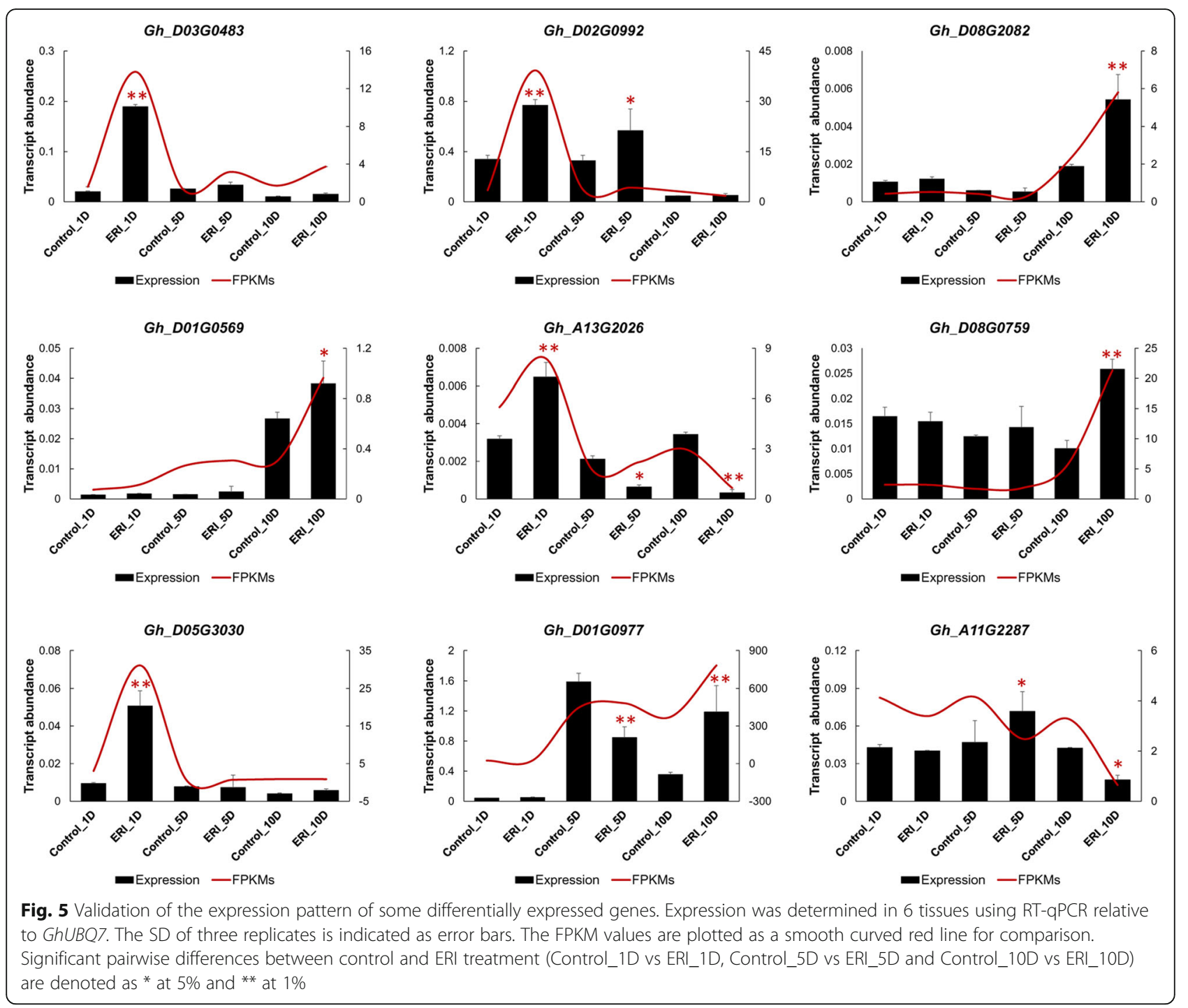

endogenous NAR were up-regulated in ERI_1D and ERI_ 5D (Fig. 6a-h). Therefore, DHK tended to accumulate during the early elongation stage (ERI_5D) since $F 3 H$ transcripts were abundant in ERI_1D tissues (Fig. 6a-h). Higher FPKM values for F3' $H$ suggested that a higher DHQ content was sustained in ERI_5D fibres, though it did not greatly differ from Control_5D (Fig. 6h). The presence of $F 3 H$ transcripts in early elongation fibre (Control_ 5D and ERI_5D) may suppress the synthesis of DHQ in ERI_5D (Fig. 6e, h). Moreover, the transcriptional activity of $F 3^{\prime} 5^{\prime} \mathrm{H}$ may also contributed to the reduced DHQ content in ERI_5D compared with ERI_1D (Fig. 6a, h).

At the maximum fibre elongation stage, flavonoid biosynthesis was suppressed, since $C H S$ the transcripts were no longer abundant in ERI_10D fibres (Fig. 6c). The higher $F 3 H$ transcript abundance was associated with significantly higher DHK content in Control_10D (Fig. 6e, i). Even though both $F 3 H$ and $F 3 ' H$ were not highly expressed in ERI_10D, ERI content did not change, since it was exogenously applied (Fig. 6b, e-i). To investigate the transcriptional modulation of the flavonoid metabolic pathway in ERI-driven elongated fibre using RNA-seq, FPKM values of putative flavonoid pathway genes were determined and plotted as a heat map (Fig. 6j). All putative $F 3^{\prime} H$ transcripts were up-regulated after 1 day in ERI-treated samples, while transcripts of a small number of putative $F 3^{\prime} H$ genes were expressed in response to ERI after 5 days (Gh_A09G1733 and Gh D03G1510) and 10 days (Gh_A05G0557 and Gh D05G0686) (Fig. 6j). In conclusion, these results show that the expression profiles of flavonoid pathway genes were related to variations in endogenous flavonoid content (Additional file 7: Table S2).

Exogenous supplementation of the flavonoid ERI resulted in the largest number of differentially expressed transcription factors (TFs) in the $M Y B$ or $M Y B$-related 


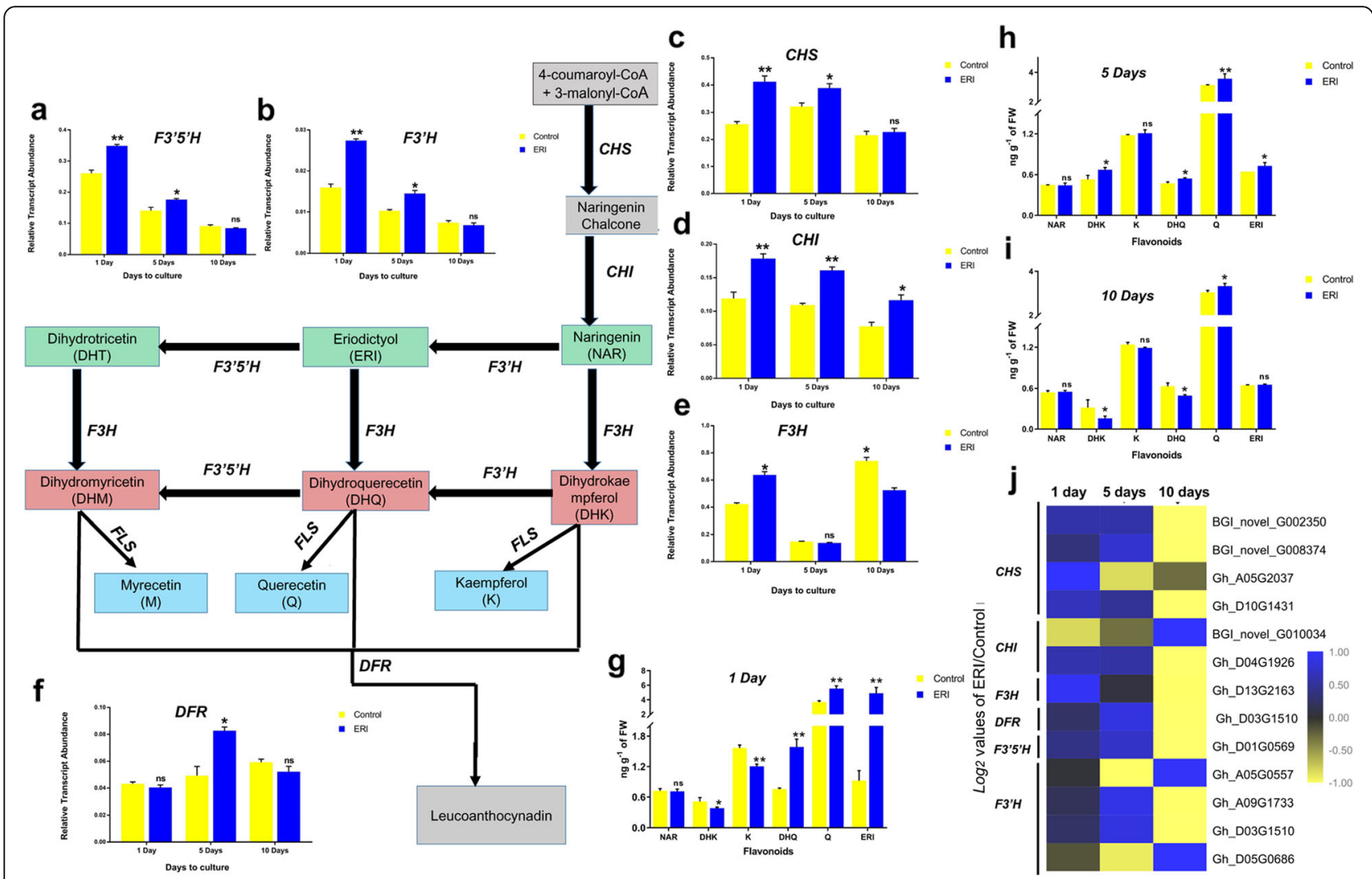

Fig. 6 The flavonoid pathway modulated in response to ERI treatment. RT-qPCR analysis of the flavonoid pathway genes $(\mathbf{a}) F 3^{\prime} 5^{\prime} H$, (b) $F 3^{\prime} H$, (c) CHS, (d) CHI, (e) F3H, and (f) DFR in 1-day ovules and in 5 and 10-day-cultured fibres for the control and ERI treatments. Quantification of different flavonoids (NAR, DHK, K, DHQ, Q and ERI) in Control and ERI samples after (g) 1 day, (h) 5 days and (i) 10 days of culture. For a-i, error bars represent the SD based on three technical repeats. Significant differences were determined and are denoted as * at 5\% and ** at $1 \%$ significance, while non-significant differences are indicated by ns. (j) FPKM values of flavonoid pathway genes derived from RNA-seq analysis are plotted in a heat map as $\log 2$ values of the ERI/control ratios

category (Additional file 9: Figure S7), and these were differentially expressed in response to ERI (Additional file 10: Figure S8). Our results showed that the expression of putative $\beta$-GALACTOSIDASE and XYLOGLUCAN ENDOTRANSGLUCOSYLASE $(X T H)$ genes was also up-regulated in developing fibres in response to ERI (Additional files 11 \& 12: Figures S9 \& S10; Additional file 7: Table S2). These results showed that key fibre growth-promoting genes were activated by ERI during fibre development.

\section{ERI and IAA together efficiently promoted cotton fibre development}

Since both IAA and $\mathrm{GA}_{3}$ were supplied in the BT medium for all ovule culture experiments, we tested whether fibre growth could be efficiently improved by ERI application in the presence of IAA or $\mathrm{GA}_{3}$ alone. Although RNA-seq based FPKM values suggested that tryptophan (KEGG00380) and diterpenoid biosynthesis (KEGG0904) pathways were differentially regulated (Additional files 13 \& 14: Figure S11 \& S12), a greater number of auxin signaling genes were up-regulated in response to ERI (Fig. 4c). Moreover, IAA quantification of ovules also revealed that IAA was available at higher concentrations even after 5 and 10 days of culture, i.e., ERI_5D and ERI_10D (Fig. 7a). To gain further insights, the expression profiles of IAA signaling components (AUX1, AUXIAA, GH3 and SAUR) were determined. The $\log _{2}$ values of ERI/Control ratios of FPKM values were visualized as a heat map, and the auxin signaling genes were found to be differentially expressed in ERI 1D, ERI_5D and ERI_10D (Fig. 7b; Additional file 7: Table S2). These results suggested that activation of the auxin signaling cascade in response to ERI might be potentially involved in the promotion of fibre growth.

Since flavonoid-induced alterations of auxin transport could also lead to temporal auxin accumulation [19], we hypothesized that auxin transport may play a role in determining the ERI-mediated fibre growth response. To test this hypothesis, the auxin transport inhibitor triiodobenzoic acid (TIBA) was added to the BT medium, and interaction effects of both IAA and $\mathrm{GA}_{3}$ with ERI 


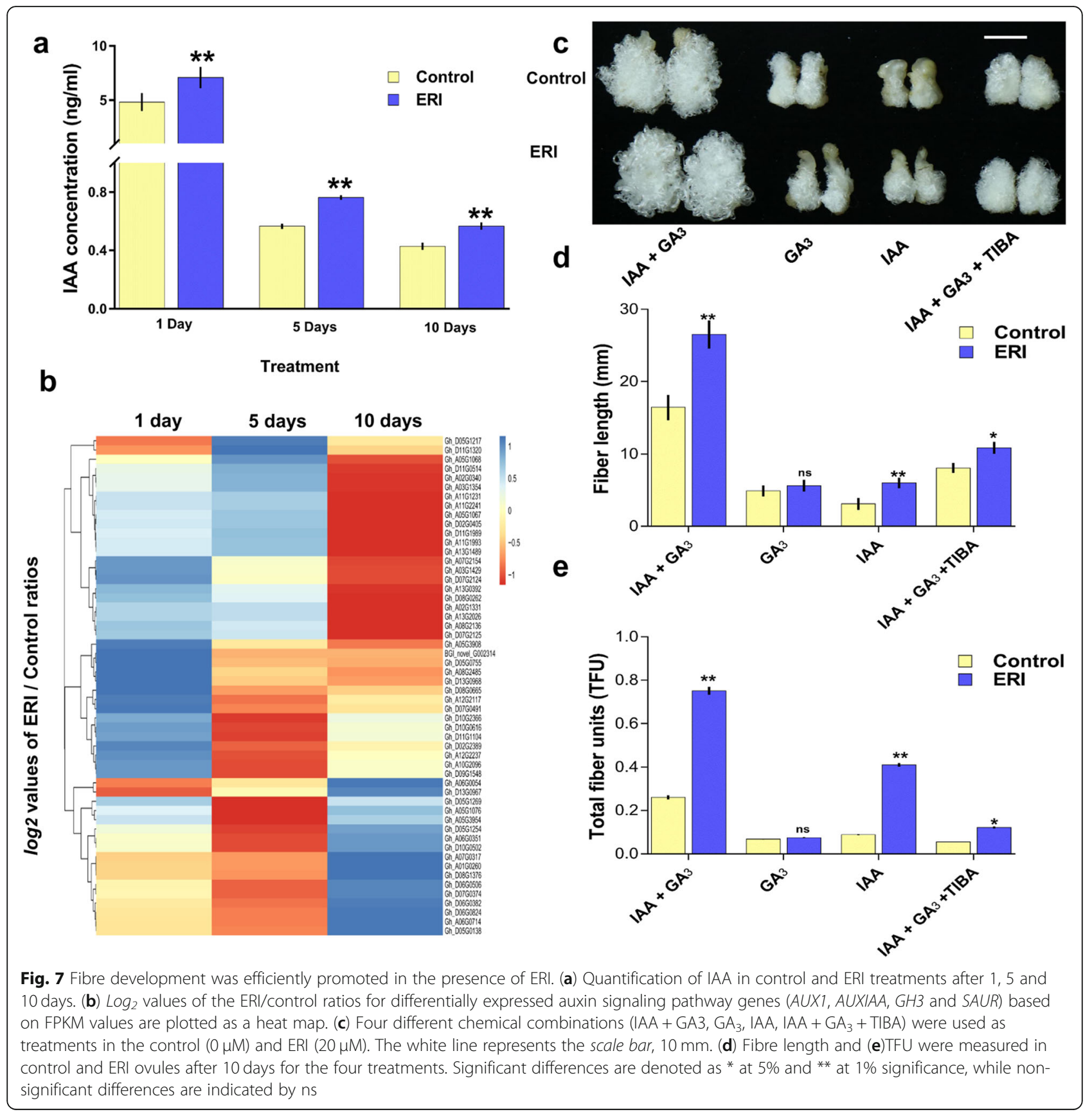

were also determined individually. After 10 days, the fibre yield was determined in ovules from four treatments: IAA $+\mathrm{GA}_{3}, \mathrm{GA}_{3}$, IAA and IAA $+\mathrm{GA}_{3}+$ TIBA.

Treatment of ovules with either IAA or $\mathrm{GA}_{3}$ could not efficiently establish fibre development even in the presence of ERI, showing that both IAA and $\mathrm{GA}_{3}$ were required for normal fibre development (Fig. 7c), and the effect of ERI on fibre elongation was not observed in the absence of IAA (Fig. 7c-e). However, ERI with IAA promoted both initiation and elongation even in the absence of $\mathrm{GA}_{3}$, though $\mathrm{GA}_{3}$ was required for maximum fibre length and TFU (Fig. 7d, e). In summary, ERI and $\mathrm{GA}_{3}$ were not sufficient to drive fibre initiation when IAA was not present, while ERI could efficiently promote fibre development in the presence of IAA $+\mathrm{GA}_{3}$ (Fig. 7ce).

To test our hypothesis concerning whether auxin transport is required for ERI-IAA-driven fibre development, we treated 0 DPA ovules with TIBA in the presence and absence of ERI for 10 days. The results showed that fibre growth was significantly reduced in presence of TIBA (Fig. 7c). However, ERI again demonstrated the 
potential to positively affect fibre length and TFU even in the presence of TIBA, suggesting the effect of ERI is at least partially independent of polar auxin transport. RNA profiling data supported the view that ERI-induced ROS homeostasis might have contributed to the enhanced fibre growth in the presence of TIBA (Additional file 6: Figure S5). These results demonstrated that ERI may work synergistically with auxin to positively modulate fibre initiation and development.

\section{Discussion}

In the flavonoid metabolic pathway, both NAR and ERI are substrates of $F 3 H$, which catalyzes the synthesis of dihydroflavanols, and F3' $H$ acts on NAR and DHK to produce ERI and DHQ (Fig. 6). The enzyme-substrate interactions have been substantiated through a biochemical analysis of $F 3^{\prime} H$ in rice grains [31]. Our results demonstrated that ERI treatment of ovules led to higher $F 3 H$ and $F 3^{\prime} H$ transcriptional activities and enhanced expression of the upstream flavonoid biosynthesis genes $\mathrm{CHS}$ and $\mathrm{CHI}$, also linked to higher endogenous ERI content (Fig. 6 a-c). This showed that the flavonoid pathway modulated a complex regulatory network to promote fibre initiation and elongation, which could be triggered by exogenous application of ERI. Our previous study reported that silencing of $F 3 H$ resulted in a short fibre phenotype in transgenic cotton, which could not be rescued in vitro by applying DHK [17]. A second study showed that NAR accumulation suppressed the effect of auxin on fibre development [32]. Therefore the available evidence suggests that NAR acts as negative modulator of cotton fibre development, while ERI acts as a positive modulator of cotton fibre. The transcriptional activity of $F 3^{\prime} H$ and its efficient translation into protein could be therefore important in determining fibre quality traits, since it is required for the biosynthesis of both ERI and DHQ (Fig. 6). Therefore, genetic modification of $F 3^{\prime} H$, not $\mathrm{F} 3 \mathrm{H}$, could provide enhanced in planta fibre growth in cotton, as this is a topic for further study.

A potential role of MYB TFs in regulating fibre development through affecting flavonoid biosynthesis has been previously described [33-35]. Over the past decade, extensive research on fibre biology has revealed many genetic components that are related to enhanced fibre development. For example, xyloglucans play a decisive role in connecting cellulose fibres and cell wall expansion during fibre elongation [36, 37]. Thus, it could be assumed that ERI triggered the fibre elongation mechanism through up-regulating MYB TFs, which somehow up-regulated the putative $\beta$-GALACTOSIDASE and XYLOGLUCAN ENDOTRANSGLUCOSYLASE (XTH) genes (Additional files 11 \& 12: Figures S9 \& S10; Additional file 7: Table S2), and all such factors constituted towards elongated fiber in ERI treated ovules.
The potential involvement of auxin in triggering fibre initiation and modulating fibre development is well established in cotton. The enhanced expression of iaaM that is related to auxin production driven by the ovulespecific promoter FLORAL BINDING PROTEIN7 (FBP7) led to increased auxin biosynthesis and significantly promoted fibre yield and quality [32]. There is compelling evidence that flavonoids can regulate auxin signaling and transport directly, via influencing the PIN efflux carriers, and auxin biosynthesis indirectly through interactions between auxin and the BR signaling pathways, i.e., through signalling crosstalk between $B R I 1$ and $A R F$ proteins [38]. In the presence of ERI, genes involved in the biosynthetic pathway towards tryptophan, required for auxin production, were up-regulated along with genes involved in auxin signaling cascade. Moreover, IAA levels were shown to accumulate upon ERI treatment (Fig. 7a; Additional file 7: Table S2). Flavonoids may also contribute to fibre elongation through modulating ROS homeostasis, since they act to scavenge ROS $[22,25]$. This ROS activity could also determine the concentration of biologically active auxin, as oxidation regulates auxin homeostasis [39]. Linked to this, our results showed that ascorbate peroxidases were up-regulated in ERI-treated ovules and fibres after 1, 5 and 10 days (Additional file 6: Figure S5).

Genetic engineering of the auxin signaling and transportation cascade has aided in promoting fibre development in cotton [40, 41]. Our results further extended the regulatory network of auxin-mediated promoted fibre development by the link with flavonoids such as ERI. Since flavonoid-induced alterations of auxin transport could lead to auxin accumulation [19, 42, 43], we hypothesized that auxin signalling is the mechanism by which ERI-mediated enhanced fibre development is controlled, and our results provided compelling evidences in support of the proposed hypothesis. We therefore concluded that the genetic and metabolic engineering of the flavonoid metabolic pathway could provide opportunities for developing high fibre yielding cotton lines with elite fibre quality parameters, through effects on auxin signalling.

\section{Conclusion}

An integrated approach was adopted to understand the role of flavonoids during cotton fibre development. The outcomes of the study demonstrated that ERI could efficiently promote fibre development. ERI showed a significant dose-dependent effect, with an optimum concentration of $20 \mu \mathrm{M}$, in promoting fibre development in terms of length and TFU. Genome wide RNA profiling data indicated that various regulatory pathways like auxin biosynthesis, auxin signalling and transportation along with the ROS homeostasis contributed to the 
enhanced fibre growth in response to ERI treatment. We also concluded that the flavonoid pathway gene expression was found linked to its content in developing fibres. Moreover, the synergistic action of ERI with auxin might be causative towards positive modulation of in vitro fibre initiation and elongation. Overall, we concluded that the genetic and metabolic engineering of the flavonoid metabolic pathway could provide opportunities for developing high quality cotton fibre.

\section{Methods}

\section{Plant materials}

Seeds of the cotton (Gossypium hirsutum) cultivar (YZ1) were collected from the Anyang Cotton Research Institute (CRI), CAAS. The seeds were grown in experimental fields or in greenhouse conditions $\left(28-35^{\circ} \mathrm{C}\right.$ day temperature, and $20-25^{\circ} \mathrm{C}$ night temperature with $\pm 16 / 8$-h light/dark cycle) at Huazhong Agricultural University, Wuhan, Hubei, China. For the ovule culture assays, flowers were harvested from the same positions on the plant in the afternoon on the day of anthesis (0 DPA) and immediately kept at $4{ }^{\circ} \mathrm{C}$. All chemicals were filter-sterilized, and flavonoids were obtained from Sigma-Aldrich.

\section{In vitro ovule culture assay and flavonoid treatments}

Two hormones, $0.5 \mu \mathrm{M} \mathrm{GA}$ and $5 \mu \mathrm{M}$ IAA, were added to BT medium, as the 'Control' for all ovule culture experiments. Initially, the exogenous concentration of each of the six flavonoids (ERI, NAR, DHQ and DHK, Q, K) was $10 \mu \mathrm{M}$, whereas five concentrations $(0,2,5,10,20$ and $40 \mu \mathrm{M})$ were used for selected flavonoids in further experiments. The in vitro ovule culture assay was performed following previously described procedures [25]. Briefly, YZ1 flowers were collected from the field or greenhouse in the afternoon at the day of anthesis $(0$ DPA), and ovaries were excised and surface-sterilized with $0.1 \% \mathrm{HgCl}_{2}$ for $15 \mathrm{~min}$ and rinsed three times with double distilled water $\left(\mathrm{ddH}_{2} \mathrm{O}\right)$. Thereafter, ovules from each ovary were dissected using sterilized forceps under aseptic conditions. Approximately $10-15$ ovules were suspended in $50 \mathrm{ml}$ control treatment flasks containing flavonoid-supplemented BT medium and, incubated in the dark without agitation at $30^{\circ} \mathrm{C}$ for 10 days. Thereafter, fibre-bearing ovules were dried on filter paper. Fibre and ovules were separated in liquid nitrogen, ground into a fine powder and stored at $-80^{\circ} \mathrm{C}$ for subsequent analysis.

\section{RNA-seq analysis and identification of DEGs}

Excised 0 DPA YZ1 ovules were cultured in BT medium without (Control) and with ERI treatment $(20 \mu \mathrm{M})$ for 10 days. The ovule culture assay was performed with three biological repeats along with three technical replicates to minimize experimental error. For genome-wide transcriptome analysis, 1-day control (Control_1D) and ERI (ERI_1D) cultured ovules were dissected under a microscope to collect embryos and immediately stored at $-80^{\circ} \mathrm{C}$ Similarly, fibres were separated from 5 and 10 day-cultured samples (Control_5D, Control_10D, ERI_ 5D and ERI_10D) and immediately stored at $-80^{\circ} \mathrm{C}$.

For transcriptomic library construction, total RNA was extracted from tissues exposed to three treatments with three replicates each (18 samples) as previously described [44]. All samples were collected for RNA-seq by Illumina HiSeq X Ten (BGI, Wuhan, China) and submitted to the NCBI Sequence Read Archive under the Bio project ID PRJNA266265 - SRX4910463 and SRX4910476. Total clean reads were mapped to the G. hirsutum (NAU assembly) cotton genome [27] using TopHat2 [45]. The reads overlapping genes were counted using HTseq-count [46]. The counts of genes were used to estimate normalization and dispersions, and they were transformed into variance stabilization data with DESeq [47]. Normalized expression was determined as FPKM (fragments per kilo bases of transcript per million mapped reads) to the reference cotton genome [27], and novel transcripts were determined using cuff compare, as described previously [48]. Differentially expressed genes (DEGs) were determined as $\geq 2$-fold change (FC) in expression. The digital expression profile of different genes was visualized by plotting the $\log 2$ values of the FPKMs ratio (ERI/Control comparisons) using the Clustvis tool [49].

\section{RNA extraction and gene expression analysis}

For gene expression analysis, fibres and ovules were collected from "Control" and "ERI" cultured samples after 1, 5 and 10 days of culture. Fibre samples were dried on filter paper, ground into a fine powder and immediately stored at $-80^{\circ} \mathrm{C}$ for total RNA extraction, as described previously [44]. Briefly, RNA was isolated from all experimental tissues using the Spectrum Plant Total RNA Kit (Sigma-Aldrich, St. Louis, MO). Synthesis of complementary DNA and RT-qPCR were performed as previously described [50]. $3 \mu \mathrm{g}$ of total RNA was used for double-stranded cDNA synthesis using the reverse transcription enzyme M-MLV (Invitrogen, Super-Script TMII), according to the manufacturer's instructions.

For the relative quantification of desired genes, RTqPCR was performed on an ABI Prism 7000 Real-Time PCR system using software (PE Applied Biosystems, USA) according to the manufacturer's instruction [25]. The RT-PCR mixture was prepared in a final volume of $20 \mu \mathrm{l}$ containing $0.4 \mu \mathrm{l}$ of each specific primer $(10 \mu \mathrm{M})$ and $10 \mu \mathrm{l}$ of $2 \times$ SYBR Green PCR Master Mix (Applied Biosystems USA). The thermal profile programme was carried out in 96-well optical RT-qPCR reaction plates (Applied Biosystems, USA) under the following conditions: $5 \mathrm{~min}$ at $95^{\circ} \mathrm{C}$ followed by 30 cycles of $35 \mathrm{~s}$ at 
$95^{\circ} \mathrm{C}$ and $35 \mathrm{~s}$ at $58^{\circ} \mathrm{C}$. Quantitative reverse transcription $\mathrm{RT}-\mathrm{qPCR}$ was performed with three biological repeats and at least three technical repeats, while the expression was normalized to GhUBQ7 and the relative transcript abundance determined using the $2^{-\Delta \Delta C t}$ method [51]. Primers of flavonoid pathway marker genes and other genes used in the RT-qPCR analysis are listed in Additional file 15: Table S3.

\section{Quantification of flavonoids and IAA}

1, 5 and 10-day-cultured ovules and fibres were washed and dried on filter paper for flavonoid quantification. Ovules from 1 day-cultured ovules, and fibres form 5 and 10 day-cultured ovules were collected from three independent repeats. All samples were ground into powder in liquid nitrogen and $750 \mu \mathrm{l}$ cold extraction buffer (methanol: water: acetic acid, 80: 19: 1, v/v/v) was added to $0.1 \mathrm{~g}$ of sample. Flavonoid quantification was performed according to previous procedures [17]. The flavonoids and IAA quantification were conducted by LC-GC/MS as previously described [52].

\section{Fibre yield measurement}

Fibre yield was determined in terms of fibre length and total fibre units (TFU). For fibre length measurements, fibre-bearing ovules were gently pulled with forceps on filter paper in a straight line. Three biological replicates were assessed, and thirty ovules were used for each replicate. The TFU was measured according to a previously described method with modifications [17]. Briefly, the cultured ovules were washed and boiled in distilled water for 5-7 min until the fibres were dispersed. The ovules were dried on filter paper and stained with $20 \mathrm{ml}$ of $0.02 \%$ Toluidine-Blue $\mathrm{O}$ solution for $30 \mathrm{~s}$. Next, the ovules were washed with running water for 2-3 min, dried on filter paper and floated in $20 \mathrm{ml}$ glacial acetic acid: ethanol: water (10: 95: 5) solution for 2-4h. The absorbance was measured in detaining solution at 624 $\mathrm{nm}$ with an Infinite 200 PRO multimode reader (Tecan). More than 36 ovules were analyzed in each technical repeat to measure TFU. All experiments were repeated five times, and each replicate constituted at least 6 technical repeats $(n \geq 6)$.

\section{Scanning electron microscopy (SEM)}

For SEM, flowers of YZ1 were picked from the same positions in the afternoon at 0 DPA, and the ovules were cultured for 1 day $(24 \mathrm{~h})$ in BT medium with Control and ERI treatments. After 1 day, the ovules were washed with $\mathrm{dd}_{2} \mathrm{O}$, immediately fixed in $2.5 \%$ glutaraldehyde and stored at $4{ }^{\circ} \mathrm{C}$ for $24 \mathrm{~h}$. Thereafter, the samples were rinsed through an ethanol series, transferred to isoamylacetate and air dried. Photographs were taken with a
JSM-6390/LV SEM (Jeol, Tokyo, Japan) to visualize fibre initials.

\section{Statistical analysis}

All experiments were repeated three times with at least three technical repeats each. For experiments in which more than two treatments were considered, ANOVA and post hoc analysis were performed in Excel to determine statistical significance. For pair-wise comparisons between two treatments, the Student's $t$ test was conducted and significance was determined at 5\%.

\section{Supplementary information}

Supplementary information accompanies this paper at https://doi.org/10 1186/s12870-019-2054-x

Additional file 1: Table S1. Statistics for the paired clean reads and read mapping for 6 treatments with 3 replications.

Additional file 2: Figure S1. Bar plots showing the expression distribution pattern in 18 samples.

Additional file 3: Figure S2. Venn plots showing pair-wise comparisons in three tissues between control and ERI treatments.

Additional file 4: Figure S3. Venn plots showing comparisons of three treatment for both (a) control and (b) ERI independently.

Additional file 5: Figure S4. Comparison of the expression profiles of novel and annotated genes between control and ERI samples.

Additional file 6: Figure S5. Heat map showing log2 values of ERI/ control ratios for ascorbate peroxidases.

Additional file 7: Table S2. Comparison of the expression profiles of novel and annotated gene between control and ERI samples.

Additional file 8: Figure S6. Heat map showing log2 values of ERI/ control ratios for BR biosynthesis pathway genes.

Additional file 9: Figure S7. Identification of transcription factors (TFs) that were differentially expressed between control and ERI.

Additional file 10: Figure S8. Expression profiles of DEG MYB transcription factors presented as log2 values of ERI/Control FPKM ratios. Additional file 11: Figure S9. Log2 values of ERI/Control FPKM ratios for putative (A) $\beta$-galactosidase genes visualized using a heatmap.

Additional file 12: Figure S10. Log2 values of ERI/Control FPKM ratios for putative xyloglucan endotransglucosylase (XTH) genes were visualized using a heatmap.

Additional file 13: Figure S11. Heat maps showing the expression profiles of IAA (tryptophan) metabolism pathway genes based on log2 values of ERI/control FPKM ratios.

Additional file 14: Figure S12. Heat maps showing the expression profiles of $\mathrm{GA}_{3}$ (diterpenoid) biosynthesis pathway genes based on $\log 2$ values of ERI/control FPKM ratios.

Additional file 15: Table S3. List of primers used in this study.

\section{Abbreviations}

DHK: Dihydrokaempferol and; DHQ: Dihydroquerectin; ERI: Eriodictyol; NAR: Naringenin; SEM: Scanning electron microscopy; TFU: Total fibre units

\section{Acknowledgements}

We acknowledge and thank Prof. Keith Lindsey from Durham University, UK for providing critical suggestions and valuable comments to improve this manuscript.

Consent to publish

Not applicable. 


\section{Authors' contributions}

AQK performed all experiments, analyzed the data and wrote manuscript. ZHL \& PCW assisted with the experiments. MMA analyzed the data and assisted with writing the manuscript, LLT and XLZ planned and designed the study and wrote and revised the manuscript. It is to mention that all authors read and approved the manuscript.

\section{Funding}

This work was funded by the National Natural Science Foundation of China (31471540). The funding body had no role in the design of the study and collection, analysis, and interpretation of data and in writing the manuscript.

\section{Availability of data and materials}

All data files generated by RNA-seq by Illumina HiSeq X Ten (BGI, Wuhan, China) have been submitted to the NCBI Sequence Read Archive under PRJNA266265 • SRX4910463 and SRX4910476.

\section{Ethics approval and consent to participate} Not applicable.

\section{Competing interests}

The authors declare that this research was conducted in the absence of any commercial or financial relationships that could be construed as a potential conflict of interest. All the authors declare that they have no competing interests.

\section{Author details}

${ }^{1}$ National Key Laboratory of Crop Genetic Improvement, Huazhong Agricultural University 430070, Wuhan, Hubei, People's Republic of China. ${ }^{2}$ Institute of Plant Breeding \& Biotechnology, MNS University of Agriculture, Multan, Pakistan.

\section{Received: 12 March 2019 Accepted: 25 September 2019} Published online: 24 October 2019

\section{References}

1. Quideau S. Flavonoids. Chemistry, biochemistry and applications. Angewandte Chemie Inter Edi. 2006;45(41):6786-7.

2. Meng C, Zhang S, Deng YS, Wang GD, Kong FY. Overexpression of a tomato flavanone 3-hydroxylase-like protein gene improves chilling tolerance in tobacco. Plant Physiol Biochem. 2015;96:388-400.

3. Zakaryan H, Arabyan E, Oo A, Zandi K. Flavonoids: promising natural compounds against viral infections. Arch Virol. 2017;162(9):2539-51.

4. Kumar S, Pandey AK. Chemistry and biological activities of flavonoids: an overview. Scient W J. 2013;2013:162750.

5. Tanaka Y, Brugliera F. Flower colour and cytochromes P450. Philos Trans R Soc Lond Ser B Biol Sci. 2013;368(1612):20120432.

6. Nix A, Paull C, Colgrave M. Flavonoid profile of the cotton plant, Gossypium hirsutum: A review. Plants. 2017;6(4)

7. Tan J, Wang $M, T u L$, Nie $Y$, Lin $Y$, Zhang $X$. The flavonoid pathway regulates the petal colors of cotton flower. PLoS One. 2013;8(8):e72364.

8. Feng $H$, Tian X, Liu Y, Li Y, Zhang X, Jones BJ, et al. Analysis of flavonoids and the flavonoid structural genes in brown fibre of upland cotton. PLoS One. 2013;8(3):e58820

9. Xiao YH, Yan Q, Ding H, Luo M, Hou L, Zhang M, et al. Transcriptome and biochemical analyses revealed a detailed proanthocyanidin biosynthesis pathway in brown cotton fibre. PLoS One. 2014;9(1):e86344.

10. Lu N, Roldan M, Dixon RA. Characterization of two TT2-type MYB transcription factors regulating proanthocyanidin biosynthesis in tetraploid cotton, Gossypium hirsutum Planta 2017;246(2):323-335.

11. Yan Q, Wang Y, Li Q, Zhang Z, Ding H, Zhang Y, et al. Up-regulation of GhTT2-3A in cotton fibres during secondary wall thickening results in brown fibres with improved quality. Plant Biotechnol J. 2018;16(10):1735-47.

12. Feng $H$, Yang $Y$, Sun S, Li Y, Zhang L, Tian J, et al. Molecular analysis of caffeoyl residues related to pigmentation in green cotton fibres. J Exp Bot. 2017;68(16):4559-69.

13. Yoo MJ, Wendel JF. Comparative evolutionary and developmental dynamics of the cotton (Gossypium hirsutum) fibre transcriptome. PLoS Genet. 2014; 10(1):e1004073.

14. Naoumkina M, Thyssen GN, Fang DD. RNA-seq analysis of short fibre mutants Ligon-lintless-1 (Li1) and -2 (Li2) revealed important role of aquaporins in cotton (Gossypium hirsutum L.) fibre elongation. BMC Plant Biol. 2015;15:65.

15. Al-Ghazi Y, Bourot S, Arioli T, Dennis ES, Llewellyn DJ. Transcript profiling during fibre development identifies pathways in secondary metabolism and cell wall structure that may contribute to cotton fibre quality. Plant Cell Physiol. 2009;50(7):1364-81.

16. Chaudhary B, Hovav R, Flagel L, Mittler R, Wendel JF. Parallel expression evolution of oxidative stress-related genes in fibre from wild and domesticated diploid and polyploid cotton (Gossypium). BMC Genomics. 2009;10:378.

17. Tan J, Tu L, Deng F, Hu H, Nie Y, Zhang X. A genetic and metabolic analysis revealed that cotton fibre cell development was retarded by flavonoid naringenin. Plant Physiol. 2013;162(1):86-95.

18. Peer WA, Murphy AS. Flavonoids and auxin transport: modulators or regulators? Trends Plant Sci. 2007;12(12):556-63.

19. Kuhn BM, Geisler M, Bigler L, Ringli C. Flavonols accumulate asymmetrically and affect auxin transport in Arabidopsis. Plant Physiol. 2011;156(2):585-95.

20. Grunewald W, De Smet I, Lewis DR, Lofke C, Jansen L, Goeminne G, et al. Transcription factor WRKY23 assists auxin distribution patterns during Arabidopsis root development through local control on flavonol biosynthesis. Proc Natl Acad Sci U S A. 2012;109(5):1554-9.

21. Brunetti C, Fini A, Sebastiani F, Gori A, Tattini M. Modulation of phytohormone signaling: a primary function of flavonoids in plantenvironment interactions. Front Plant Sci. 2018;9(1042):1042.

22. Peer WA, Cheng Y, Murphy AS. Evidence of oxidative attenuation of auxin signalling. J Exp Bot. 2013;64(9):2629-39.

23. Peer WA, Blakeslee JJ, Yang H, Murphy AS. Seven things we think we know about auxin transport. Mol Plant. 2011:4(3):487-504.

24. Kim HJ, Hinchliffe DJ, Triplett BA, Chen ZJ, Stelly DM, Yeater KM, et al. Phytohormonal networks promote differentiation of fibre initials on preanthesis cotton ovules grown in vitro and in planta. PLoS One. 2015;10(4): e0125046.

25. Guo K, Du X, Tu L, Tang W, Wang P, Wang M, et al. Fibre elongation requires normal redox homeostasis modulated by cytosolic ascorbate peroxidase in cotton (Gossypium hirsutum). J Exp Bot. 2016;67(11):3289-301.

26. Qian SH, Hong L, Cai YP, Gao JS, Lin Y. Effects of light on in vitro fibre development and flavonoid biosynthesis in green cotton (Gossypium hirsutum). Acta Societ Botan Polon. 2016;85(2).

27. Zhang T, Hu Y, Jiang W, Fang L, Guan X, Chen J, et al. Sequencing of allotetraploid cotton (Gossypium hirsutum L. acc. TM-1) provides a resource for fibre improvement. Nat Biotechnol. 2015;33(5):531-7.

28. Marino D, Dunand C, Puppo A, Pauly N. A burst of plant NADPH oxidases. Trends Plant Sci. 2012;17(1):9-15.

29. Suzuki N, Miller G, Morales J, Shulaev V, Torres MA, Mittler R. Respiratory burst oxidases: the engines of ROS signaling. Curr Opin Plant Biol. 2011; 14(6):691-9.

30. Zhu J-Y, Sae-Seaw J, Wang Z-Y. Brassinosteroid signalling. Development (Cambridge, England). 2013;140(8):1615-20.

31. Park S, Choi MJ, Lee JY, Kim JK, Ha SH, Lim SH. Molecular and biochemical analysis of two rice Flavonoid 3'-Hydroxylase to evaluate their roles in flavonoid biosynthesis in rice grain. Int J Mol Sci. 2016:17(9).

32. Zhang M, Zheng X, Song S, Zeng Q, Hou L, Li D, et al. Spatiotemporal manipulation of auxin biosynthesis in cotton ovule epidermal cells enhances fibre yield and quality. Nat Biotechnol. 2011;29(5):453-8.

33. $\mathrm{Pu} L$, Li Q, Fan $X$, Yang W, Xue Y. The R2R3 MYB transcription factor GhMYB109 is required for cotton fibre development. Genetics. 2008;180(2):811-20.

34. Huang W, Sun W, Lv H, Luo M, Zeng S, Pattanaik S, et al. A R2R3-MYB transcription factor from Epimedium sagittatum regulates the flavonoid biosynthetic pathway. PLoS One. 2013;8(8):e70778.

35. Liu B, Zhu Y, Zhang T. The R3-MYB gene GhCPC negatively regulates cotton fibre elongation. PLoS One. 2015;10(2):e0116272.

36. Lee J, Burns TH, Light G, Sun Y, Fokar M, Kasukabe Y, et al. Xyloglucan endotransglycosylase/hydrolase genes in cotton and their role in fibre elongation. Planta. 2010;232(5):1191-205.

37. Naoumkina M, Hinchliffe DJ, Fang DD, Florane CB, Thyssen GN. Role of xyloglucan in cotton (Gossypium hirsutum L.) fibre elongation of the short fibre mutant Ligon lintless-2 (Li2). Gene. 2017;626:227-33.

38. Tian H, Lv B, Ding T, Bai M, Ding Z. Auxin-BR interaction regulates plant growth and development. Front Plant Sci. 2017:8:2256.

39. Zhang J, Peer WA. Auxin homeostasis: the DAO of catabolism. J Exp Bot 2017;68(12):3145-54 
40. Wang MY, Zhao PM, Cheng HQ, Han LB, Wu XM, Gao P, et al. The cotton transcription factor TCP14 functions in auxin-mediated epidermal cell differentiation and elongation. Plant Physiol. 2013;162(3):1669-80.

41. Zhang M, Xiao Y, Zeng J, Pei Y. PIN-formed protein, a door to reveal the mechanism for auxin-triggered initiation of cotton fibre. Plant Signal Behav. 2017;12(5):e1319031.

42. Yin R, Han K, Heller W, Albert A, Dobrev PI, Zažímalová E, Schäffner AR. Kaempferol 3-O-rhamnoside-7-O-rhamnoside is an endogenous flavonol inhibitor of polar auxin transport in Arabidopsis shoots. New Phytol. 2014; 201(2):466-75.

43. Steenackers W, Klíma P, Quareshy M, Cesarino I, Kumpf RP, Corneillie S, Araújo P, Viaene T, Goeminne G, Nowack MK, Ljung K, Friml J, Blakeslee JJ, Novák O, Zažímalová E, Napier R, Boerjan W, Vanholme B. Cis-Cinnamic acid is a novel, natural auxin efflux inhibitor that promotes lateral root formation. Plant Physiol. 2017;173(1):552-65.

44. Zhu LF, Tu LL, Zeng FC, Liu DQ, Zhang XL. An improved simple protocol for isolation of high quality RNA from Gossypium spp. suitable for CDNA library construction. Acta Agron Sin. 2005;31:1657-9.

45. Kim D, Pertea G, Trapnell C, Pimentel H, Kelley R, Salzberg SL. TopHat2: accurate alignment of transcriptomes in the presence of insertions, deletions and gene fusions. Genome Biol. 2013;14(4):R36.

46. Anders S, Pyl PT, Huber W. HTSeq--a Python framework to work with highthroughput sequencing data. Bioinformatics. 2015;31(2):166-9.

47. Anders S, McCarthy DJ, Chen Y, Okoniewski M, Smyth GK, Huber W, et al. Count-based differential expression analysis of RNA sequencing data using $R$ and bioconductor. Nat Protoc. 2013;8(9):1765-86.

48. Trapnell C, Roberts A, Goff L, Pertea G, Kim D, Kelley DR, et al. Differential gene and transcript expression analysis of RNA-seq experiments with TopHat and cufflinks. Nat Protoc. 2012;7(3):562-78.

49. Metsalu T, Vilo J. ClustVis: a web tool for visualizing clustering of multivariate data using principal component analysis and heatmap. Nucleic Acids Res. 2015;43(W1):W566-70.

50. Tu LL, Zhang XL, Liu DQ, Jin SX, Cao JL, Zhu LF, et al. Suitable internal control genes for RT-qPCR normalization in cotton fibre development and somatic embryogenesis. Chin Sci Bull. 2007:52(22):3110-7.

51. Schmittgen TD, Livak K. Analyzing real-time PCR data by the comparative $C_{T}$ method. Nat Protoc. 2008:3:1101-8.

52. Liu H, Li X, Xiao J, Wang S. A convenient method for simultaneous quantification of multiple phytohormones and metabolites: application in study of rice-bacterium interaction. Plant Methods. 2012;8(1):2.

\section{Publisher's Note}

Springer Nature remains neutral with regard to jurisdictional claims in published maps and institutional affiliations.

Ready to submit your research? Choose BMC and benefit from:

- fast, convenient online submission

- thorough peer review by experienced researchers in your field

- rapid publication on acceptance

- support for research data, including large and complex data types

- gold Open Access which fosters wider collaboration and increased citations

- maximum visibility for your research: over $100 \mathrm{M}$ website views per year

At $\mathrm{BMC}$, research is always in progress.

Learn more biomedcentral.com/submissions 